\title{
Towards the Big Picture: Enriching 3D Models with Information Visualisation and Vice Versa
}

\author{
M. Sedlmair, K. Ruhland, F. Hennecke, A. Butz, S. Bioletti, and C. O'Sullivan \\ BMW Group Research and Technology, Munich, Germany \\ Media Informatics Group, University of Munich, Germany \\ Preservation and Conservation Department, Trinity College Dublin, Ireland \\ Graphics Vision and Visualisation Group, Trinity College Dublin, Ireland \\ \{michael.sedlmair, fabian.hennecke\}@bmw.de, andreas.butz@ifi.lmu.de, \\ \{kerstin.ruhland, carol.osullivan, bioletts\}@tcd.ie
}

\begin{abstract}
Most information visualisation methods are based on abstract visual representations without any concrete manifestation in the "real world". However, a variety of abstract datasets can indeed be related to, and hence enriched by, real-world aspects. In these cases an additional virtual representation of the $3 \mathrm{D}$ object can help to gain a better insight into the connection between abstract and real-world issues. We demonstrate this approach with two prototype systems that combine information visualisation with $3 \mathrm{D}$ models in multiple coordinated views. The first prototype involves the visualisation of in-car communication traces. The 3D model of the car serves as one view among several and provides the user with information about the car's activities. LibViz, our second prototype, is based on a full screen 3D representation of a library building. Measured data is visualised in overlaid, semi-transparent windows to allow the user interpretation of the data in its spatial context of the library's 3D model. Based on the two prototypes, we identify the benefits and drawbacks of the approach, investigate aspects of coordination between the 3D model and the abstract visualisations, and discuss principals for a general approach.
\end{abstract}

Key words: Information Visualisation, 3D Models, Multiple Coordinated Views

\section{Introduction}

While we were applying Information Visualisation methods to real-world scenarios in our earlier projects [17-19], we observed that in many cases relevant data does not exist in a purely abstract form, as assumed by many information visualisation approaches. In addition to the abstract data, there is often some connection to real-world entities such as cars or buildings. We refer to such data as hybrid, multivariate data because it consists of an abstract part as well as a direct reference to some real-world behaviour or entity. We postulate that this type of data is available for large number of real-world scenarios and therefore merits further investigation. 
Traditional approaches are mostly designed for either abstract or real-world data and can be categorised as being either Information (InfoVis) or Scientific (SciVis) Visualisation. While SciVis basically deals with the representation of measured data with the help of virtual 3D objects [20], InfoVis concentrates on abstract data and representations $[3,20]$. However, the boundary between these two areas is not always easy to identify, as demonstrated by our own experience with real-world applications. Therefore, comprehensive solutions and combined approaches are usually required.

In fact, there are already some existing systems that visualise such hybrid information. For instance, many InfoVis applications are based on a combination of abstract representations together with colour coded and coordinated 2D maps to clarify the correlation of geospatial and abstract information. In SciVis, on the other hand, virtual 3D representations of real-world objects are enhanced by descriptive, often simply textual information. These concepts have proven helpful in gaining insights into the correlations between abstract and objectrelated information.

In this paper, starting from an InfoVis point of view, we focus on situations where abstract data is also related to a specific, self-contained representation of the real world. For such cases, we propose the enrichment of abstract visualisations with virtual 3D representations of the underlying real-world entity, by visualising them simultaneously in a multiple coordinated view (MCV) system.

We propose two different approaches and demonstrate them with prototypical applications. First, we present CarComViz, a visualisation system for in-car communication data. CarComViz extends a previously implemented, abstract MCV visualisation [19] with an additional 3D model of the car and combines both approaches in parallel displayed views. CarComViz supports automotive engineers and diagnostic experts in navigating and getting insight into highly complex and abstract traced data from in-car networks. Up to $15 \mathrm{~K}$ messages per second are distributed over 13 bus systems in one car, and the size and complexity of this dataset requires multivariate visualisation support. The network data has an inherent relationship to the car itself, as it is the technical cause or effect of activities within the vehicle.

We also present $L i b V i z$, a visualisation of multivariate measured data from the Old Library in Trinity College Dublin, introduced in [17]. In LibViz, a user can navigate through the virtual library, while visualising additional information about measured, location dependent data, such as dust levels, humidity information or window conditions. The application combines a wide variety of data sources to provide preservation specialists with a general overview and to allow insight into complex aggregate information. Unlike CarComViz, the representation of the library is displayed in full screen mode and presents the central view of the application. Additional abstract visualisations are overlaid in semitransparent windows and are dynamically coordinated with the 3D model. This approach is common in 3D gaming, for instance. 


\section{Related Work}

Multiple Coordinated Views (MCV) are commonly used to visualise highly complex, multivariate and multidimensional data [11]. Many InfoVis applications use this technique to combine abstract visualisations such as scatter plots, parallel coordinates visualisation or treemaps. One major benefit of MCV is the potential to reveal relationships within the data [1]. Therefore, many tools elucidate relationships between the abstract part of the data and its reference to realworld entities by integrating $2 \mathrm{D}$ representations of these entities within an MCV system. Such applications typically depict medical data [12] or geospatial information $[16,7]$. In contrast, SciVis systems focus more on technical artefacts and the direct mapping of measured data onto a 3D representation of the artefact. Additional 2D Views are used to display legends and additional textual information.

In recent years, the trend has been towards abolishing the somewhat artificial boundary between InfoVis and SciVis $[14,15]$. While the "separate but equal" approach was appropriate in some cases, it has become increasingly clear that SciVis and InfoVis approaches must converge in order to gain deeper insights into interrelated, complex areas. Kosara et al. presented a MCV framework linking SciVis and InfoVis, using 2D scatter plots for abstract data and 3D scatter plots for artefact representation [9]. Similar approaches can be found in the Weave [5] and VizCraft [4] systems. Such spatial data can also be represented by solid 3D representations. Husoy and Skourup [6] presented an approach using a 3D model of an industrial plant as the user interface for a control system. Within the plant's 3D model, the user has direct access to data and information integrated in the model. Butkiewicz et al. [2] developed an interactive MCV analysis tool that combines two 3D views with a heat map for chronological visualisation of changes to an urban environment. One major benefit of their tool is that it can demonstrate relationships between the multivariate data and real urban model by using the $3 \mathrm{D}$ presentation of the landscape together with the $2 \mathrm{D}$ heat map.

Last but not least, our approach is also inspired by $3 \mathrm{D}$ game technology. The enrichment of virtual 3D worlds with additional 2D data is very common in this community. Textual and graphical 2D overlays are used to show additional information such as game status, avatar behaviour or maps, and support navigation and orientation [10]. However, there is one fundamental difference between using $2 \mathrm{D}$ overlays in $3 \mathrm{D}$ games and in task driven visualisation approaches like ours. For gaming, 2D elements are viewed only as a necessary evil, decreasing immersion in order to show additional information. However, for visualisation purposes, the $2 \mathrm{D}$ elements are an essential contribution towards the goal of gaining greater insights.

\section{Goal and Requirements}

As discussed above, 3D representations of real-world entities enriched with abstract information have been proposed before. However, in this paper we do not 
focus on a direct mapping of information onto a 3D model, as commonly applied in SciVis applications. Rather, we investigate how 3D models can be integrated into MVC systems in order to improve the understanding of semantic connections between abstract data and real-world entities. In doing so, the 3D model serves less as a carrier of complex information, but more as a visual connection of the data to reality.

To use the proposed combination of abstract visualisation and 3D models, it is necessary that the underlying data set fulfils some basic criteria - it must be: multivariate (it consists of several variables) and hybrid (it is abstract in one variable and connected to the real world in another). Obviously, for purely abstract data without any connection to real-world entities, or real-world data alone, our approach is not appropriate.

In reality, multivariate, hybrid datasets are often complex and hard to understand. Representing the information in a MCV system allows the complexity to be divided [1]. The abstract part of the data can be visualised via established or novel approaches from InfoVis, while one or more additional views can show the real-world aspects of the data using a virtual 3D representation. Thus, visual correlations between the views can provide insight into the connections between abstract and real-world information.

\section{Two Example Prototypes}

Below, we will present two of our prototype systems, each showing a different MVC approach to combining 3D models with information visualisations. CarComViz (an extension of [19]) is based on an equitable side-by-side presentation of views, whereas LibViz [17] uses a centralised full-screen 3D model with overlaid information windows.

\subsection{CarComViz}

CarComViz is an application to visualise in-car communication traces. Such recorded data from in-car communication networks is a good example of multivariate, hybrid data. The core data is of an abstract nature and represents, in simple terms, signals and messages sent between functions. However, these functions in turn are implemented in so-called electronic control units (ECUs), which are the hardware components containing the function. Hence, they are obviously related to the real-world vehicle by the position in which they are installed. Furthermore, certain signals cause physical effects such as moving a window or switching on the light. Automotive diagnostic experts record the data directly from the vehicle via a hardware interface and specific recording tools. The traces contain a sequence of up to $15 \mathrm{~K}$ messages per second, and therefore become very large. The messages include information about the sending functional blocks (which in turn implies the sending ECUs), various encapsulated signals and further detailed information such as timestamps. 
Currently used analysis software such as Canalyzer ${ }^{1}$ or Tracerunner ${ }^{2}$ are very powerful, but mostly text based tools. However, the lack of visualisation makes it hard to understand the correlations within the data. To achieve a deeper understanding of the bus communication, it is necessary to gain more insight into the timing and causal connections between messages and real behaviour. Therefore, we designed a tool in which the user can visually browse traces and see detailed information about messages, while monitoring activities and the current physical state of the vehicle.

Design: CarComViz is designed to support visual analysis during offline data diagnosis. It is based on three views (cf. Fig. 1):

The Autobahn View is the central, abstract view of this application. It can be seen as an offspring from the scatter plot. It is a customized method built on a metaphor of a crowded motorway. A bus system is visualised as separate superior blocks, the motorway. Every bus transports messages from different ECUs. This transportation is represented by a horizontal bar, which would be a lane according to the motorway comparison. The lane contains black rectangles, which represent every message sent by the ECU through the bus to another ECU. Instead of showing the sent messages, this view can also be used to visualise sent signals. Interaction within the view is based on zooming and panning. Starting with an overview, the user can zoom into interesting parts of the trace and can horizontally as well as vertically pan around at each zoom level.

A traditional List View represents detailed information about the data in a textual line-up. The user can interactively select items from the Autobahn View and show the corresponding detailed information in this view, which can be additional information about exact timing, encapsulated signals or the hexadecimal raw data. We found that the classical textual representation was still the best way to show this detailed information.

In addition to these abstract views (cf. [19]), a novel 3D Model View depicts a virtual model of the vehicle of interest. Real behaviour, such as moving the windows, turning on the lights or accelerating wheels, is presented by animation. Navigating through time in the Autobahn View causes resulting activities to occur in the 3D model, depending on the particular message content. Additionally, the user can configure different perspectives of the car and view them in a "picture within a picture" style. By dynamically adjusting the transparency of the car's components, the focus of the different perspectives can be set to be very flexible. The perspective views can be freely repositioned within the window and dynamically toggled by clicking on them. The selected perspective view is then swapped into the main stage where it can be configured and navigated. Furthermore, the user can initialise supplemental 2D overlays, which are directly connected to a specific component of the car via a semitransparent arrow. Both mechanical components, e.g., a wheel, and electronic components, such as ECUs or bus systems, can be thus enhanced. The $2 \mathrm{D}$ overlay can show a specific user defined value of interest, such as the actual speed or bus load that changes over

\footnotetext{
${ }^{1}$ www. canalyzer.de

2 www.tracerunner.de
} 


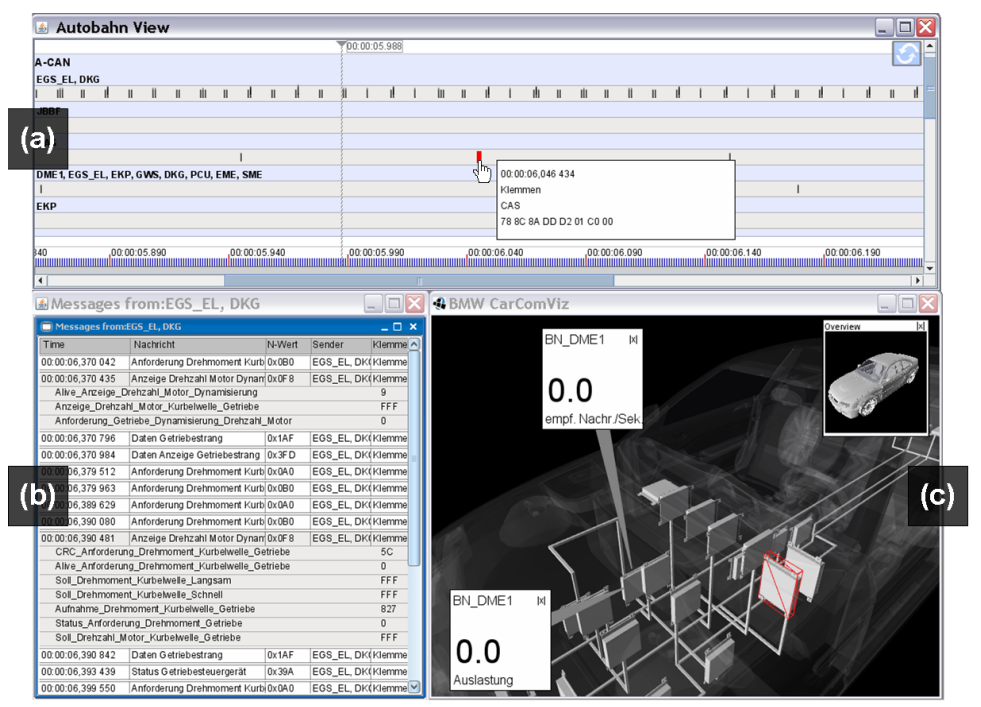

Fig. 1. CarComViz: (a) Autobahn View, (b) List View, (c) 3D Model View

time. Interface configuration tasks (creating perspective views or 2D overlays, adjusting transparency) are set manually in a detached settings dialogue.

Evaluation: We conducted a qualitative user study with five automotive domain experts, focusing on the additional 3D View. The participants were confronted with tasks of differing complexity. The tasks dealt with the recognition of car states and the identification of causal relationships between a 3D model and abstract network information, such as "which message caused the door to open?" With our user study, we did not aim to benchmark ourselves with respect to existing industrial analysis tools. Our goal was to clearly focus on evaluating the specific effects of integrating a virtual 3D model into the analysis process.

All participants acknowledged the overall benefit of using the 3D model in the combined and correlated representation with the abstract $2 \mathrm{D}$ visualisations, which is encouraging. Exclusively abstract representations were not effective in linking the information to real-world behaviour, while the exclusively 3D representation was less useful for analysing in-car communication traces. The expertise of the user, however, has a significant effect, in that the more expert (s)he is, the less 3D model visualisation is necessary. The visual correlations between the views were understood very well. Also, obvious state transitions such as opening a door were easily identified, with only small scale changes such as activating the indicator being difficult to detect. For such subtle effects, other techniques and solutions for 3D highlighting should be investigated.

\subsection{LibViz}

LibViz [17] is a tool to assist the Preservation and Conservation Department in preserving the collection of the Old Library in Trinity College Dublin. The 


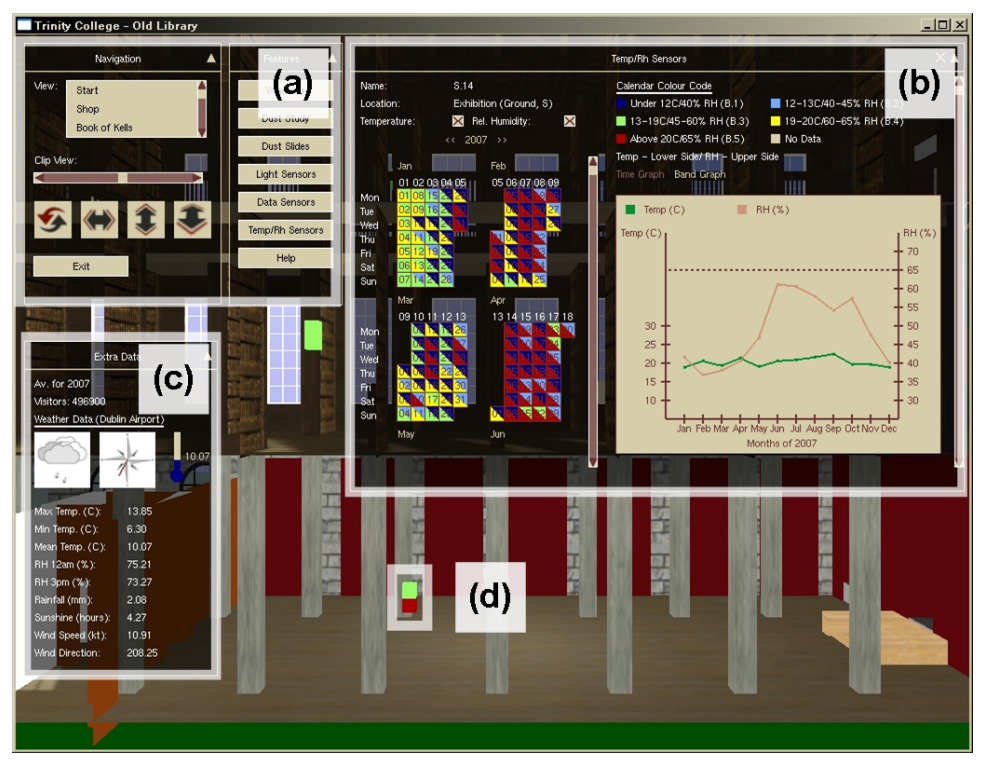

Fig. 2. LibViz: (a) Widget for global functions, (b) Detail widget, (c) Extra data widget, (d) Sensor within the 3D model

application visualises a variety of data recorded in the Library. So far, it provides information about windows, dust, temperature and relative humidity, along with additional data about visitor statistics and external weather data. This multivariate, hybrid data is visualised using appropriate types of information visualisation methods and can be explored using a $3 \mathrm{D}$ virtual reconstruction of the Old Library. This representation serves to elucidate structural and environmental correlations and allows the user to compare and assess the relationships between the various datasets in a spatial context.

Design: In LibViz, semi-transparent 2D widgets overlay a 3D virtual reconstruction of the Old Library. Since the main use of the application is the visualisation of recorded data, the 3D model of the Library is kept reasonably simple. The user can navigate freely inside the model by using either virtual buttons provided in the interface, or mouse/keyboard navigation similar to computer game controls.

Two initial 2D widgets contain global functions such as navigation controls, a clipping slider and a list of feature buttons to activate the various data visualisations. The purpose of the other widgets is to show the actual data in the form of abstract information visualisations. To exemplify the interaction between the 3D model and the 2D widgets, we describe the temperature and relative humidity feature (cf. Fig. 2). Activating this feature draws neutrally coloured cubes at the position where the sensors are located inside the library. These sensors can be inspected by navigating inside the $3 \mathrm{D}$ environment or by using the clipping slider from the global function widget to remove layers of the building. Furthermore, 
an opened widget lists all available sensor names. To select a specific sensor, the user chooses one from the list or by clicking on the 3D representation of the sensor. Both activities open the detail widget and the extra data widget. Furthermore the sensor cubes are coloured depending on the average data over the selected time frame for temperature (in the lower half of the cube) and relative humidity (in the upper half). By choosing a sensor, the date is set to the year of the last recorded sensor dataset.

The detail widget holds a Calendar View [21] and a Graph View. The average temperature of each calendar day defines the colour code of the initial Calendar View. Using check boxes, the user can decide if the calendar and the graph should show temperature, relative humidity or both. This view allows the user to identify patterns and thereby possibly hazardous situations. The second view of this widget contains a time graph. In addition to the internal environmental conditions, the extra data widget contains visitor numbers and external weather data. A bar graph of the percentage of visitors and weather icons allows an overview of the available data to be visualised. The time graph of the detail widget, the extra data widget and the coloured sensor cubes in the 3D environment are linked to the Calendar View. Selecting a specific year, month, week or day changes the representation and content of these three components. This enables the user to examine the data from different points of view and to compare the various influencing factors. The data can be set in connection with the sensor position inside the building and the external weather data. Different sensors can be directly compared with each other inside the 3D environment.

The time component is suited to the dust accumulation feature as well. Dust sensors are distributed in the Library's Long Room and are visualised as spheres that are assigned the colour code of the last measurement. The detail widget of this feature provides a time slider over the measurement dates. Sliding through time changes the sensor's colour and removes or adds sensors according to their appearance.

Evaluation: A user study was conducted to determine a) whether the design of the application met the needs of its target users and b) how effectively the users were at completing assigned tasks using the system. There were eleven participants, of whom five were library employees and six were computer graphic scientists. In the first trial, the users had to complete four tasks, followed by a questionnaire and a final trial with slightly modified tasks. The given tasks were designed to test users' ability to find the information they were asked for and to direct their attention to specific details in the application. Using the example of the temperature and relative humidity feature, the user was instructed to locate a specific sensor and to record its colour code and internal and external temperature on a specific day. The usage questionnaire focused on extracting the correct information using the application. The users found it easy to work with the $2 \mathrm{D}$ representation such as the Calendar View or time graphs. While the majority of the users had no problems selecting the correct sensor from the 3D environment, they nevertheless preferred selecting the sensor from a list. Overall, feedback was very positive. 


\section{Towards a General Understanding}

During the successful implementation and evaluation of these two prototype systems, some general underpinning principles began to emerge. The combination of $3 \mathrm{D}$ visualisation with $2 \mathrm{D}$ abstract InfoVis elements clearly combines the benefits of both worlds without sacrificing too much of either. We now develop these ideas further and propose some general principles for hybrid visualisation applications.

\subsection{Combining the Views}

We proposed two different approaches to combine 3D models with information visualisation in a MCV system: Equal Views and 3D Model with Overlays. These approaches illustrate the trade-off between allocating screen space and accepting overlaps. CarComViz, for instance, is based on the approach of Equal Views. One or more views of the 3D model serve as parallel views within a MCV system. The views are more or less equal and dynamically divide the screen space without overlaying each other. The technique is commonly used in the area of MCV applications. LibViz, on the other hand, uses the approach of a central 3D model with overlays. This approach is more common in 3D gaming. The approach can be seen as a mixture of sequential and parallel representation of views. The $3 \mathrm{D}$ model of the entity forms the centre of the visualisation and is shown in the entire application window or in full screen. While this was not necessary for CarComViz, LibViz benefits from a full screen representation of the 3D model because it allows an adequate and parallel presentation of all sensors within their spatial context. This improves the overall comprehension of correlations between the high number of sensors. A smaller representation would either cause a loss of presentable sensors, if zoomed in, or a loss of information perceptibility, if zoomed to an entire view. To show the abstract information, additional $2 \mathrm{D}$ visualisations are overlaid on demand and are dynamically linked to the 3D model. In LibViz, we accepted the drawback of overlapping because of the reason mentioned above. To counteract the effects we did two things: Firstly, the overlaid windows were set semitransparent so that a certain degree of context is retained. Secondly, the windows can be dynamically minimised to show the entire, non-overlapped 3D model view on demand.

To summarise, both approaches are equally valid, but a full screen representation should only be used when the application truly justifies it. It may be the case that the problem of overlapping can only be partially addressed using interaction and transparency. Furthermore, the decision about which approach to take depends on where the user's focus should be. A 3D Model with Overlay representations will draw the main attention to the $3 \mathrm{D}$ model and relegate the abstract visualisations to the status of additional information sources. On the

other hand, the Equal View approach does not make any inherent prediction of where the focus should be concentrated. The user can decide whether to start with abstract visualisations and use the $3 \mathrm{D}$ model view for context, or vice versa. 


\subsection{Linking the Views}

There are several different approaches to linking views in MCV applications. We identify two general ways that are usable for our target applications:

Item Linking: Brushing and Linking is a common interaction technique in the area of MCV [8]. Usually it is used to highlight identical data items in different views. Also, elements that reveal a clear relationship, e.g., messages sent by a certain ECU and the ECU itself, can be graphically bonded. Combining 3D models with abstract visualisations provides one aspect of linking and brushing that goes beyond the conventional use of highlighting identical elements. In addition to connecting equal elements over view boundaries, it allows spatial (3D-)positions to be highlighted according to a selected abstract item. Spatial awareness can be achieved due to the fact that one (or more) representations of the items are shown in their real-world entities' context. In CarComViz, for instance, hovering over a message element in the Autobahn view will cause the bounding box of its encapsulating ECU to be highlighted in the 3D model view. The highlighting colours in both views are the same and the user can immediately make the connection.

Semantic Linking: Semantic Linking refers to the fact that an interaction, e.g., a selection or navigation, in an abstract view causes some actual behaviour in the 3D model. CarComViz, in particular, used this specific form of linking the views. Navigating over time in the Autobahn view initiates a sequence of activities in the car's 3D model. For instance, the vehicle's front door opens, it closes again and the wheels begin to move. This would correspond to a forward navigation in a situation where the driver enters the car, starts the engine and moves off. The equivalent backwards navigation would cause a reset of actions so that the status of the vehicle stays correct. In general, this yields two novel forms of coordinating views:

1. Showing activities: A specific data chunk causes some real-world activity of the entity. The behaviour can be visualised via animation and the user can reproduce the connection between the activity and abstract information.

2. Status of entity: In the case of time dependent data, the 3D model of the entity allows the user to see the actual state of the entity at each point in time. Navigating over the time line updates the status of the 3D model.

Both aspects can help the user to get a broader insight into complex, multivariate, hybrid data by clarifying the entity's real-world context.

\subsection{Navigating the Views}

During our user studies we observed that some users experienced difficulties with navigating within the 3D models. Such problems only occurred with novice users, unlike expert users who had some 3D gaming experience. In LibViz, we therefore integrated two different $3 \mathrm{D}$ navigation variants to counteract this. It turned out that the button controls were especially helpful for novice users, whereas the mouse/keyboard navigation mode was preferred by more expert users. Initially, 
this problem is a generic 3D issue. By combing $2 \mathrm{D}$ and $3 \mathrm{D}$ elements we additionally impose the need to mentally switch between different control modes in 3D and 2D. However, we did not observe that users had a particular problem with this.

\section{Discussion}

There is a high user demand for $3 D$ model based visualisations. That is a fundamental insight gained during our user-centred development approaches to LibViz and CarComViz. Some of the most common statements were: "3D models just look good", "That's fun to use and really intuitive" or "With the model I can easily communicate things, even to my boss...". It would be disingenuous to state that such subjective considerations were not a major motivating factor for our work. However, we do temper this enthusiasm with a disclaimer: $3 D$ model based visualisation is appealing, but in order to make it truly useful in practical applications, it should be easy to control and enriched with additional $2 D$ representations to visualise multivariate, hybrid data.

Initially, there should be a clear spatial aspect to the data, which is meaningful for the user. Otherwise, a 3D model visualisation may look nice but provide no beneficial insights. A simple 2D representation, even though it is not as graphically attractive for the user, will provide better performance. Conversely, once the added value of using 3D has been proven, it is not sufficient in most cases to simply enrich the $3 \mathrm{D}$ models with complex abstract information. The abstract dimensions of the data cannot be directly mapped into the 3D real-world space, as the amount of data will exceed the user's perceptual capacity. For example, showing all messages in a vehicle running over all buses in real time would lead to cognitive overload.

However, if these prerequisites are satisfied, there are several benefits of using $3 \mathrm{D}$ models in combination with information visualisation tools. As we can see above, the basic benefit of our approach is that we can combine abstract information with real entities or put it in its real spatial context. Additionally, the virtual 3D model provides capabilities that real-world objects do not. For example, we can easily turn a car around to see its bottom side, or take slices off a library to show its interior. This also enables us to directly support pre-attentive perception to focus and highlight elements or activities of interest.

\section{Conclusion and Future Work}

This paper documents our experience with combining approaches of InfoVis and 3D model representations by means of two example desktop applications. After describing the design of these two systems, we then took a step back in order to identify common underlying principles and proposed guidelines for the design of future systems.

The main difference between the two approaches taken in our two examples is the way in which they combine 3D and InfoVis elements, and how the user 
can navigate between 2D and 3D respectively. Both approaches are justifiable for their respective applications. They each have relative benefits and drawbacks, and therefore we have provided guidelines about when to use them. The main commonality between both approaches is the fact that they both manage to establish a real-world reference of abstract data quite clearly. They allow, for example, to navigate a time line in the abstract view and thereby essentially add a 4th (temporal) dimension to the (coordinated) 3D view as an additional freely controllable parameter (in contrast to just playback). Events in the data can clearly be linked to physical motions in the 3D model (and vice versa), and data elements can be related to 3D positions in the real world. Both of these connections would be impossible to draw without the combination of abstract $2 \mathrm{D}$ and realistic $3 \mathrm{D}$ visualisations.

There are still challenges and questions which need to be examined, for instance: "How can elements be suitably highlighted in the 3D model and what are the relative benefits of various techniques?", "How can we apply useful, approach-specific automatic changes in the $3 \mathrm{D}$ view, e.g., automatically set transparencies or support automatic camera navigation?", "Which is the most appropriate form of 3D navigation for novice users?", or "Are there any specific effects of context switching between abstract 2D and modelled 3D?". Another major challenge will be to evaluate our systems further. It is particularly difficult to objectively measure the enhancement of understanding that our visualisations deliver (in fact, this is a challenge faced in general for InfoVis, cf. [13]).

We hope that with these insights, we have stimulated a systematic approach to combining $2 \mathrm{D}$ visualisations of abstract data with realistic $3 \mathrm{D}$ visualisations. Combining these methods, as described in this paper, can allow the user to understand the bigger picture more easily, without sacrificing the strengths of the respective techniques used - a clear case of the sum being greater than the parts. In our future work, we will generate more examples of this combination and hope to be able to achieve an even tighter coupling and eventually provide a quantitative evaluation of the benefits.

\section{References}

1. M. Baldonado, A. Woodruff, and A. Kuchinsky. Guidelines for using multiple views in information visualization. In Proceedings of the working conference on Advanced visual interfaces, pages 110-119. ACM Press New York, NY, USA, 2000.

2. T. Butkiewicz, R. Chang, Z. Wartell, and W. Ribarsky. Visual Analysis and Semantic Exploration of Urban LIDAR Change Detection. In Computer Graphics Forum, volume 27, pages 903-910. Blackwell Publishing, 2008.

3. S. Card, B. Shneiderman, and J. Mackinlay. Readings in information visualization. Morgan Kaufmann Publishers San Francisco, Calif, 1999.

4. A. Goel, C. Baker, C. Shaffer, B. Grossman, W. Mason, L. Watson, and R. Haftka. VizCraft: A Problem-Solving Environment for Aircraft Configuration Design. Computing in Science and Engineering, pages 56-66, 2001.

5. D. Gresh, B. Rogowitz, R. Winslow, D. Scollan, and C. Yung. WEAVE: a system for visually linking 3-D and statistical visualizations, applied to cardiac simulation 
and measurement data. In Proceedings of the conference on Visualization'00, pages 489-492. IEEE Computer Society Press Los Alamitos, CA, USA, 2000.

6. K. Husøy and C. Skourup. 3d visualization of integrated process information. In NordiCHI, pages 497-498, 2006.

7. M. Jern, S. Johansson, J. Johansson, and J. Franzen. The GAV Toolkit for Multiple Linked Views. In Coordinated and Multiple Views in Exploratory Visualization, 2007. CMV'07. Fifth International Conference on, pages 85-97, 2007.

8. D. Keim. Information Visualization and Visual Data Mining. IEEE Transactions on Visualization and Computer Graphics, pages 1-8, 2002.

9. R. Kosara, G. Sahling, and H. Hauser. Linking scientific and information visualization with interactive 3D scatterplots. In Proc. WSCG Short Communication Papers, pages 133-140, 2004.

10. C. Maple, R. Manton, and H. Jacobs. The use of multiple co-ordinated views in three-dimensional virtual environments. In $I V$, pages 778-784, 2004.

11. C. North and B. Shneiderman. Snap-together visualization: a user interface for coordinating visualizations via relational schemata. In Proceedings of the working conference on Advanced visual interfaces, pages 128-135. ACM New York, NY, USA, 2000.

12. C. North, B. Shneiderman, and C. Plaisant. Visual Information Seeking in Digital Image Libraries: The Visible Human Explorer. Information in Images, 1997.

13. C. Plaisant. The challenge of information visualization evaluation. In Proceedings of the working conference on Advanced visual interfaces, pages 109-116. ACM New York, NY, USA, 2004.

14. T. Rhyne. Does the Difference between Information and Scientific Visualization Really Matter? IEEE Computre Graphics and Apllications, pages 6-8, 2003.

15. T. Rhyne, M. Tory, T. Munzner, M. Ward, C. Johnson, and D. Laidlaw. Information and Scientific Visualization: Separate but Equal or Happy Together at Last. In Proceedings of the 14th IEEE Visualization 2003 (VIS'03). IEEE Computer Society Washington, DC, USA, 2003.

16. S. Roth, P. Lucas, J. Senn, C. Gomberg, M. Burks, P. Stroffolino, J. Kolojejchick, and C. Dunmire. Visage: a user interface environment for exploring information. Proceedings of Information Visualization, pages 3-12, 1996.

17. K. Ruhland, S. Bioletti, M. Sedlmair, and C. O'Sullivan. Libviz: Data visualisation of the old library. In Proceedings of the 14th International Conference on Virtual Systems and Multimedia dedicated on Digital Heritage, Oct. 2008.

18. M. Sedlmair, W. Hintermaier, K. Stocker, T. Büring, and A. Butz. A dual-view visualization of in-car communication processes. In Proceedings of the 12th International Conference on Information Visualization, July 2008.

19. M. Sedlmair, B. Kunze, W. Hintermaier, and A. Butz. User-centered Development of a Visual Exploration System for In-Car Communication. In 9th International Symposium on Smart Graphics, SG 2009, Salamanca, Spain, May 28-30, 2009. Proceedings, May 2009.

20. M. Tory and T. Mller. Human factors in visualization research. IEEE Transactions on Visualization and Computer Graphics, 10:72-84, 2004.

21. J. van Wijk and E. van Selow. Cluster and calendar based visualization of time series data. In Proceedings of the 1999 IEEE Symposium on Information Visualization, page 4. IEEE Computer Society Washington, DC, USA, 1999. 